\title{
Testing a theoretical prediction for bar formation in galaxies with bulges
}

\author{
Sandeep Kumar Kataria ${ }^{1,2}$, Mousumi Das ${ }^{1}$, and Sudhanshu Barway ${ }^{1}$ \\ ${ }^{1}$ Indian Institute of Astrophysics, Koramangla, Bangalore 560034, India \\ e-mail: skkataria.iit@gmail.com \\ 2 Indian Institute of Science, Bangalore 560012, India
}

Received 18 January 2020 / Accepted 9 June 2020

\begin{abstract}
Earlier studies have shown that massive bulges impede bar formation in disk galaxies. Recent $N$-body simulations have derived a bar formation criterion that depends on the radial bulge force in a galaxy disk. We use those simulations to show that bars can form only when the force constant $F B<0.13$, where $F B$ depends on the ratio of the bulge force to the total force of the galaxy at twice the disk scale length $2 R_{d}$. In this article, we test this theoretical prediction using observational data obtained from the literature. Our sample consists of 63 barred galaxies with a wide range of Hubble classes taken from the $\mathrm{S}^{4} \mathrm{G}$ catalog; for the galaxies that we include, bulge, disk, and bar decomposition has been carried out. We find that $92 \%$ of our sample galaxies satisfy the condition $F B<0.13$ for bar formation in galaxies and hence agree with the bar formation criterion given by the simulations.
\end{abstract}

Key words. galaxies: bulges - galaxies: spiral - galaxies: statistics

\section{Introduction}

Early studies have shown that a massive halo component can prevent the formation of bars in disk galaxies (Ostriker \& Peebles 1973; Hohl 1976). The presence of a massive halo makes a disk kinematically hot and prevents bar instabilities from forming. It has also been shown that a massive central bulge component prevents bar formation as it introduces inner Lindblad resonances (Sellwood 1980) and cuts the feedback mechanism such that swing amplification no longer works (Toomre et al. 1981). Apart from bulge mass, the effect of halo densities on bar formation has also been found to be important; the corresponding criterion that depends on halo to disk mass ratios is known as the Efstathiou, Lake \& Negroponte criterion (hereafter; ENL criterion) (Efstathiou et al. 1982). However, the application of the ENL criterion for bar formation is limited because the criterion is only based on two-dimensional disk simulations. Also the stellar velocity dispersion was not included and instead of a live dark matter halo, a rigid one was used to model the halo potential. These studies were limited by the availability of computational resources and hence rigid halos were used. Later studies have also shown with various models that the presence of a rigid halo does not allow the exchange of angular momentum between the disk and halo particles, which is important for the formation and evolution of bars (Athanassoula 2008).

Other studies that have examined the effect of galaxy mass distributions on bars include the effect of the central mass concentration of galaxies on bars (Norman et al. 1996; Shen \& Sellwood 2004; Athanassoula et al. 2005; Debattista et al. 2006; Kataria \& Das 2019). These studies claim that bars become weaker with the increasing central mass concentration (CMC) until the mass is a few percent of the disk mass, at which point the bar dissolves. Massive compact CMCs (Shen \& Sellwood 2004) have been found to dissolve bars as they produce chaos in the large scale phase space region occupied by the bar, while lower mass CMCs make bars weak by producing chaos in the smaller phase space regions of the bar.

Recent 3D $N$-body simulations of isolated disk galaxies with live dark matter halos have shown that both bulge mass and bulge concentration have a strong effect on bar formation timescales in disk galaxies (Kataria \& Das 2018) (hereafter KD2018). The criterion depends on the ratio of the force due to the bulge and that due to the total galaxy; the forces are measured at the disk scale length $R_{d}$, and the criterion is given by $F B=\frac{G M_{\text {bulge }}}{R_{d} V_{\text {tot }}^{2}}$. The above mentioned study suggested that if $F B>0.35$ (Force due to bulge), the disk does not form a bar when evolved under isolation.

Observational studies have also shown that a correlation exists between bulges and bars, and a possible preference for bars to form in bulge dominated systems, although this may be an observational bias as bulge dominated spiral galaxies are often easier to spot (Sheth et al. 2008; Skibba et al. 2012). In fact, the results of large optical surveys show that the bar fraction decreases with increasing bulge brightness (Barazza et al. 2008; Aguerri et al. 2009). There is also a correlation between the CMC within bulges in barred galaxies and bar ellipticity (Das et al. 2003, 2008). Also, from observations of early to late type spiral galaxies along the Hubble sequence, it is clear that bulges become less prominent (Binney \& Tremaine 2008). Furthermore, some observations show that the bulge to disk mass ratio decreases from early to late type spiral galaxies (Laurikainen et al. 2007; Graham \& Worley 2008). The correlation of bars with bulge to disk flux ratios (B/D) is clearly seen in Laurikainen et al. (2007) where the B/D ratio is smaller for early type barred spiral galaxies compared to early type non-barred spiral galaxies. Most of these studies indicate that bars should be less common in spirals with massive bulges, and their presence makes it harder to form bars in their disks. 
However, the issue is more complicated than just bulge mass. For example studies such as Díaz-García et al. (2016) show that bars are preferentially found in galaxies in which the mass is more centrally concentrated. This suggests that bulge mass may not be the only important factor, but bulge mass concentration may also play an important role in the evolution of disk structure and bar formation in galaxy disks.

Both observations (Barazza et al. 2008; Aguerri et al. 2009) and simulations (KD2018) indicate that massive bulges do not allow bars to form easily in the disks of galaxies and that bulge concentration may also play a role. In this regard the bar formation criterion $F B=0.35$, determined from simulations of disk galaxies by KD2018 is an important theoretical prediction that can be tested with observations of barred galaxies. The criterion is written in terms of parameters that can be easily obtained from observations and includes the effect of both bulge mass and concentration.

We will also briefly discuss the different types of bulges, their formation mechanism, and properties since we have compared our numerical results with observations. There has been several discussions about the type of bulges in simulations compared to observations (Athanassoula 2005; Fisher \& Drory 2008; Erwin et al. 2015). Athanassoula (2005) suggested that there are three types of bulges, which are classical bulges, boxy/peanutshaped bulges, and disky pseudo bulges. Classical bulges are rounder objects and kinematically hotter, they are similar to elliptical galaxies, and are thought to be formed by major mergers (Kauffmann et al. 1993; Baugh et al. 1996; Hopkins et al. 2009; Naab et al. 2014), multiple minor mergers (Bournaud et al. 2007; Hopkins et al. 2010), accretion of smaller satellites (Aguerri et al. 2001), and monolithic collapse of a primordial cloud (Eggen et al. 1962). Boxy/peanut shaped bulges are vertical, thick systems and rotationally dominated in comparison to classical bulges. They are thought to be formed by disk instability during secular evolution (Kormendy \& Kennicutt 2004), vertical heating of the bar due to buckling (Combes et al. 1990; Raha et al. 1991; Martinez-Valpuesta et al. 2006), or heating of the bar due to vertical resonances (Pfenniger \& Norman 1990). Disky pseudo bulges are flattened system like an exponential disk in the nuclear region. They are thought to be formed by the inward pulling of gas along the orbits and the consequent star formation (Heller \& Shlosman 1994; Regan \& Teuben 2004). We have discussed the comparison between bulges of simulations (KD2018) and observations used in this article in Sect. 3.

In this article, our aim is to obtain the value of $F B$ for a sample of barred galaxies in order to test the bar formation criterion mentioned above. To make a fair comparison, we have limited the sample of galaxies with bulge-to-total mass ratios similar to that of the simulated models in KD2018. The article is organized as follows. Section 2 summarizes the bar formation criterion. In Sect. 3, we describe the sample data, its analysis, and the comparison of observational bulges to simulated ones. Section 4 is devoted to results and discussion. A summary of this work is presented in Sect. 5.

\section{Revised bar formation criterion}

The simulations of KD2018 showed that both bulge mass and bulge concentration play an important role in inhibiting bar formation in disk galaxies. The bulge concentration is defined as the ratio of the effective bulge radius to disk scale length. The study included two types of disk models with increasing bulge masses called MA and MB, which differ in bulge concentrations. The disk scale lengths for models $\mathrm{MB}$ are almost twice

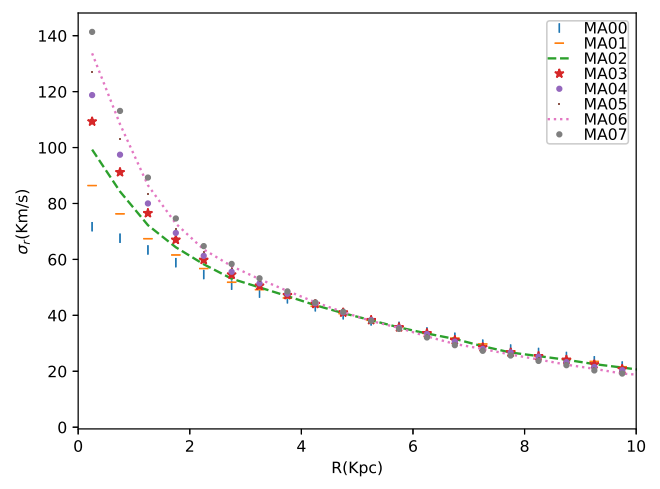

Fig. 1. Radial velocity dispersion for all MA models with increasing bulge masses. The increasing bulge mass order is from MA00 to MA07; MA00 is without a bulge and MB07 has the highest bulge mass.

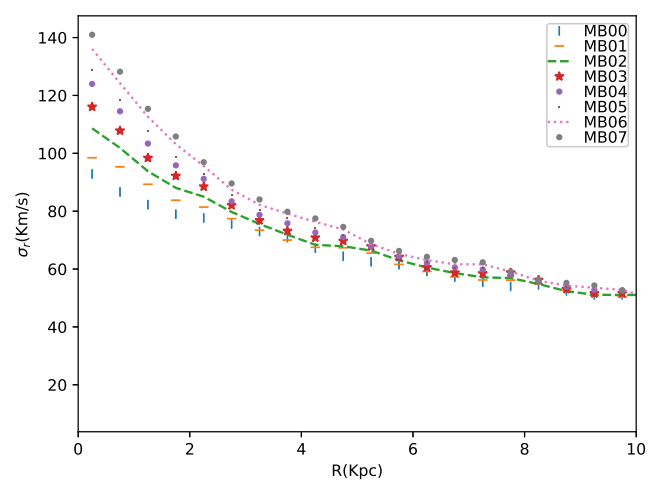

Fig. 2. Radial velocity dispersion for all MB models with increasing bulge masses. The increasing bulge mass order is from MB00 to MB07; MB00 is without a bulge and MB07 has the highest bulge mass.

that of models MA. Hence, MB models represent less concentrated galaxies compared to models MA (more details are given in KD2018). The study showed that as bulge mass increased, bars could not form in the disks. This is because a massive bulge makes a disk kinematically hotter as the central gravitational potential becomes deeper with increasing bulge mass. Figures 1 and 2 show this clearly; we find that as the bulge mass increases for MA and MB type models, it leads to a gradual increase in central velocity dispersion.

In KD2018, the models have bulge to disk mass ratios that vary from 0 to 0.7 or bulge to total galaxy mass ratios that vary from 0 to 0.41 . The study showed that if the ratio of force due to the bulge component and that due to the total galaxy potential $(F B)$ exceeds a value of 0.35 , the bar instability will not develop in the disk. The relation is given by

$$
F B=\frac{F_{b}}{F_{\text {tot }}}=\frac{G M_{\text {bulge }}}{R_{\mathrm{d}} V_{\text {tot }}^{2}} .
$$

Here $M_{\text {bulge }}$ is the mass of the bulge component, $R_{\mathrm{d}}$ is the disk scale length, and $V_{\text {tot }}$ is the total rotational velocity at disk scale length. The study also showed that the bulge mass that inhibits bar formation is lower for models with dense bulges (MA) compared to models with less dense bulges (MB).

However, KD2018 used the initial values (i.e., before evolution) of disk scale length and rotation velocity to calculate the value of FB in the criterion. However, the disk scale length and rotation curve change significantly as a disk forms a bar and secularly evolves until it reaches 9.79 Gyr. Hence, we have revised 
the calculations of FB using the final disk scale length and rotation curves to deduce FB values. In the revised version of the FB criterion we calculated the rotation velocity values at a radius that is twice that of the disk scale length. This is because this radius falls in the flat rotation curve of disk galaxies. We find that if FB exceeds a value of 0.13 , the disk does not support a bar. Therefore, the revised value of the criterion is given by

$F B=\frac{F_{b}}{F_{\text {tot }}}=\frac{G M_{\text {bulge }}}{2 R_{\mathrm{d}} V_{\text {tot }}^{2}}<0.13$

Here $M_{\text {bulge }}$ is the mass of the bulge component, $R_{\mathrm{d}}$ is the disk scale length, and $V_{\text {tot }}$ is the total rotational velocity at twice the disk scale length.

The above formula can be applied to observations fairly easily as long as the bulge luminosity, disk scale length and galaxy rotation velocities are known for a sample of galaxies.

\section{Data}

The first two parameters in Eq. (2), $M_{\text {bulge }}$ and $R_{d}$, can be obtained from the 2D bulge disk decomposition of a galaxy. For this purpose, we have used the bulge disk decomposition of barred galaxies provided by Salo et al. (2015). These 2D decompositions were done using $3.6 \mu \mathrm{m}$ images from the Spitzer Survey of Stellar Structure in Galaxies $\left(\mathrm{S}^{4} \mathrm{G}\right)$ using the GALFIT software (Peng et al. 2002, 2010). The study by Salo et al. (2015) provides the bulge, disk, and bar parameters of a sample of 103 barred galaxies along with reliable estimates of their bulge Sersic index and the bulge-to-total light ratio $(\mathrm{B} / \mathrm{T})$.

The other parameter in the bar formation criterion is the rotation velocity at twice the disk scale length, $V_{\text {tot }}$. To determine this parameter we used the Hyperleda ${ }^{1}$ database (Paturel et al. 2003; Makarov et al. 2014). This constraint further reduces our sample size to 94 because of the unavailability of rotation curves. For some of the individual sources we have used HI line widths at full width at half maximum (FWHM), also called the W50 value in the literature, to determine $V_{\text {tot }}$. It is a measure of the total width at half the peak value of the HI line and is approximately twice the disk flat rotation velocity in a galaxy. These W50 values have been further corrected for galaxy inclination angle taken from Hyperleda database to obtain final flat rotation velocities $V_{\text {tot. }}$. These rotation velocities correspond to the outer region of galaxies. However, the $F B$ calculation needs rotation velocities at twice the disk scale lengths and so this approximation may result in lower values of the $F B$ constant in some galaxies. A discussion for this limitation is given in Sect. 4 .

In the simulations presented in KD2018, an upper limit of 0.41 for the bulge-to-total mass ratio was used. To obtain the bulge mass for our sample of galaxies, we used mass-to-light $(\mathrm{M} / \mathrm{L})$ ratio values of 0.5 (Lelli et al. 2016) to convert the bulge luminosity to bulge mass. The bulge luminosity is taken directly from a 2D decomposition of the galaxies (Salo et al. 2015), which derived the bulge luminosities as a fraction of the total galaxy luminosity. Figure 3 shows the distribution of our sample data with respect to the bulge-to-total mass ratio. Here, the vertical dashed line denotes the upper limit of the bulge-to-total mass used in simulations (KD2018). In order to have a fair comparison, we removed the galaxies that are outside the upper limit of the bulge-to-total ratio used for simulated galaxies. This further reduces our sample to 87 galaxies. As nearly face-on as well as edge-on galaxies will lead to errors in de-projecting the rotation curves, we have only included galaxies that have inclination

\footnotetext{
1 http://leda.univ-lyon $1 . \mathrm{fr} /$
}

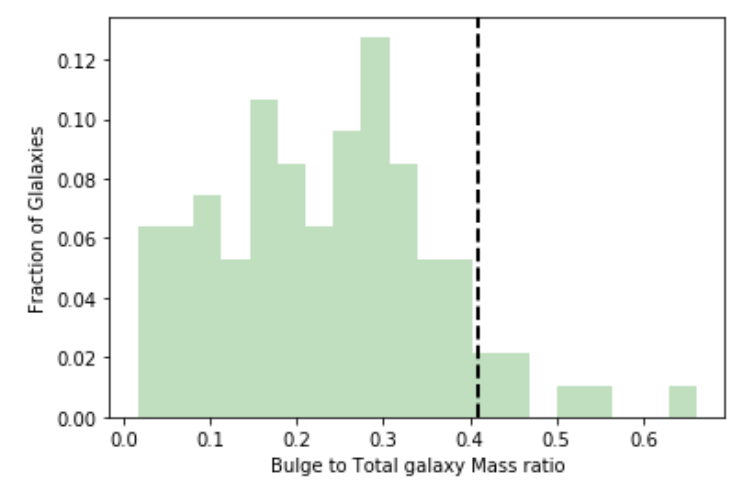

Fig. 3. Distribution of bulge-to-total disk mass for the sample galaxies. Here the vertical dashed line represents the upper limit of this ratio in the simulations of KD2018.

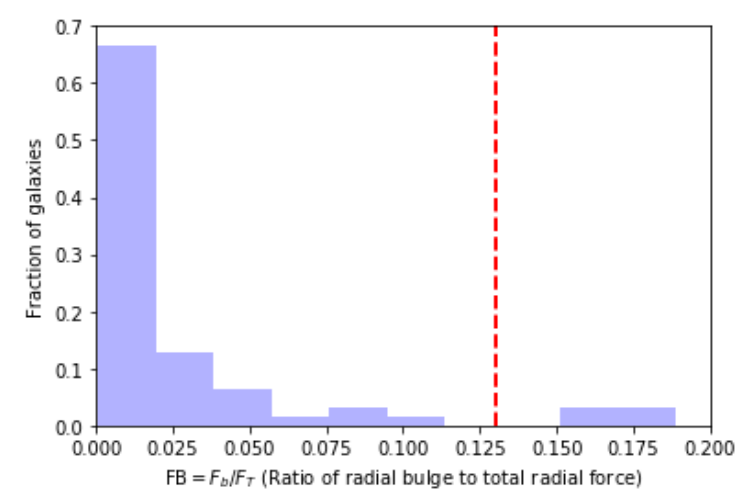

Fig. 4. Distribution of galaxies given according to the ratio FB, i.e., ratio of radial bulge force to total radial force.

angles less than $80^{\circ}$ and larger than $30^{\circ}$. This restricts our final sample to 63 galaxies to include in the study. Unless otherwise mentioned, we have used Wilkinson Microwave Anistropy Probe cosmology (Bennett et al. 2013) to convert angular distances to physical distances.

To compare our results of barred galaxies with an unbarred sample, we also compiled the 2D decomposition parameters of an unbarred galaxy sample (Salo et al. 2015) to calculate the FB criterion. We obtained 113 unbarred galaxies after putting similar constraints to those we applied for the barred galaxies discussed above in this section: bulge-total mass fraction, inclination angle, and availability of rotation curve.

Data from Salo et al. (2015) used for this study define bulges based on their Sersic indices and include both classical $(n>2)$ and boxy/peanut bulges or pseudo bulges $(n<2)$ (Fisher \& Drory 2008). In the KD2018 simulations, the galaxy models in the beginning of the simulations have bulges with hernquist profiles (Yurin \& Springel 2014), which are typically classical in nature. As the galaxy models evolve, the buckling of the bar introduces additional boxy bulges (B/P) (Athanassoula 2005). Therefore the KD2018 models contain composite bulges at the end (Erwin et al. 2015), which have both classical as well as boxy bulges. However, the $F B$ criterion from KD2018 includes only the initial classical bulge mass determined from the beginning of simulations. The measured observational $F B$ criterion (Salo et al. 2015) may have a mixture of boxy/peanuts, disky pseudo bulges, and classical bulges. Hence the observed $F B$ values will be an overestimate compared to the simulated ones. 


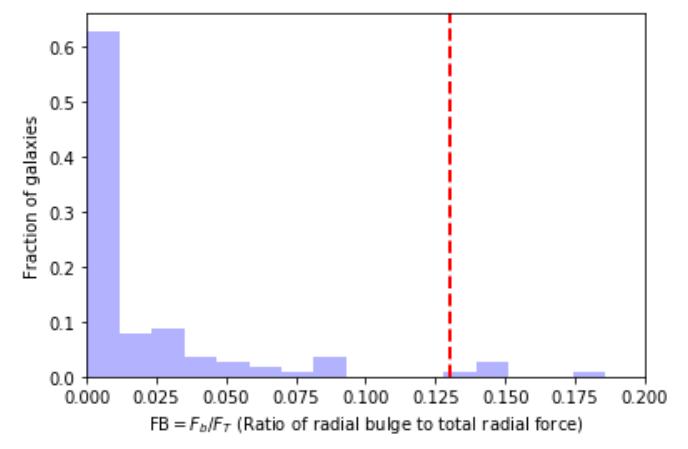

Fig. 5. Distribution of unbarred galaxy according to the ratio $F B$ parameter defined in Eq. (2).

\section{Results}

Once the observational data for the galaxy sample had been obtained, we calculated the force constant $F B$ for our sample using the bulge masses, the disk scale lengths, and the flat rotational velocities for each galaxy. In Fig. 4 we have plotted the histogram of the $F B$ parameter for the barred galaxy sample. We find that around $92 \%$ of the galaxies in our sample lie below the value of 0.13 (Fig. 4). This confirms that the value of $F B=0.13$ represents an upper bound on the fractional radial bulge force that allows bar formation (KD2018).

The barred galaxies with FB values larger than 0.13 are ES0 509-026, NGC 3489, NGC 4355, and NGC 6278. Of these, ESO 509-026 is interacting with the neighboring galaxy IC 4249 (Telles \& Terlevich 1995) and hence does not agree with the isolated evolution constraint of the bar formation criterion ( $F B$ parameter). Galaxy NGC 3489 is also part of the Leo group (Watkins et al. 2014) and is interacting with other galaxies within the group; hence it does not agree with the constraint of isolated galaxy evolution. For NGC 4355, the HI detection may be spurious (Beckmann et al. 2006) as the HI detection has been confused with galaxy VV655 (Thomas et al. 2002). Galaxy NGC 6278 is in the phase of early stage merger activity (BarreraBallesteros et al. 2015) and hence again the isolated galaxy condition for $F B$ criteria is not satisfied for this galaxy.

In Fig. 5 we have plotted the $F B$ criterion value for the unbarred galaxy sample. We find that surprisingly most of the unbarred galaxies are below the upper limit of bar formation, $F B=0.13$. This is because the presence of a massive bulge is a necessary condition for inhibiting bar formation in a galaxy disk but it is not a sufficient one. Therefore our bar formation criterion is a necessary condition for a disk to be stable but not a sufficient one as there are a few other factors that are beyond the scope of the simulations (KD2018).

The main difference between the simulation models and the observations of galaxies is that in the $F B$ criterion models, the disk velocity dispersion is mainly determined by the bulge mass and the initial model parameters. However, in real galaxies there are several possible methods by which a disk can increase its velocity dispersion, such as: (1) tidal interactions with satellite galaxies (Elmegreen et al. 1995); (2) when a galaxy passes through cluster potential (Valluri 1993); (3) the accretion of a minor galaxy by a major galaxy, which heats the stars in the major galaxy disk (Qu et al. 2011); and (4) secular evolution of a bar; if a bar has dissolved and it is comparable to the bulge size, it will heat up the disk and bulge (Guo et al. 2020). Thus a disk may be stable against bar formation despite the presence of an allowed bulge mass $(F B<0.13)$ as suggested by our criterion because of the higher velocity dispersion (Athanassoula 2008). This has an important implication for unbarred galaxies that satisfy $F B<0.13$. It may mean that their disks are kinematically hot compared to similar galaxies that do not satisfy this criterion.

\section{Discussion}

The HI line widths $W_{50}$ used in this study give an approximate estimate of two times the flat rotation velocity of the galaxy disk after correcting for inclination. However, the criterion given by Eq. (2) requires the rotation curve at disk scale length $2 R_{\mathrm{d}}$. In general $V_{\text {tot }}$ at $2 R_{\mathrm{d}}$ will be less than $\frac{1}{2} W_{50}$. Hence, our estimates of $F B$ are a lower limit to the values of Eq. (2). For example, in the case of NGC 4569, we initially used a flat rotation velocity $V_{\text {tot }}$ of $185 \mathrm{~km} \mathrm{~s}^{-1}$. However, the rotation curve shows that $V_{\text {tot }}$ at $2 R_{\mathrm{d}}$ corresponds to $179.6 \mathrm{~km} \mathrm{~s}^{-1}$ (Sofue et al. 1999). Thus, in NGC 4569 the $F B$ parameter changes from 0.022 to 0.024 . However, both values satisfy the bar formation criterion $(F B<0.13)$. Similarly, if we assume that the rotation curve values at $2 R_{\mathrm{d}}$ are lower by $25 \%$ compared to the maximum value of the rotation curve, which is typically the case for most of the galaxies (Sofue et al. 1999), our criterion will still be valid for $90 \%$ of the sample galaxies.

It will be interesting to explore this bar formation criterion $(F B)$ for our Galaxy, the Milky Way, which is a barred spiral galaxy (Gerhard et al. 2002). Theoretical modeling of photometric and kinematic data (Binney \& Tremaine 2008; McMillan 2011; Bovy \& Rix 2013; Licquia et al. 2016) predicts that the disk scale length $R_{\mathrm{d}}$ value of the Milky Way varies from 2 to $3 \mathrm{kpc}$. The rotation curve was obtained from McGaugh (2018). There is uncertainty over the presence of a classical bulge in our galaxy (Bland-Hawthorn \& Gerhard 2016). We calculated the classical bulge mass that is required to satisfy $F B<0.13$ at twice the disk scale length and found that it varies from 0.44 to $0.76 \times 10^{10} M_{\odot}$. This agrees with the upper limit for the allowed classical bulge mass in the Milky Way, which is around $0.84 \times 10^{10} M_{\odot}$ (Portail et al. 2015), and is certainly a much smaller mass in the central region of Milky Way.

The simulations of KD2018 show that the central mass concentration in a disk galaxy makes it kinematically hotter, that is, it increases the velocity dispersion of disk stars. This can adversely affect bar formation as a bar will not form in a hot disk. We can see that barred galaxies obey the criterion defined by Eq. (2). On the other hand, we see that the unbarred galaxy sample does not seem to follow the bar formation criterion because it is a necessary condition for bar formation but is not a sufficient one. The criterion is insufficient due to factors other than the presence of a bulge that affect disk velocity dispersion, such as tidal interaction and satellite merging, which make disks hotter.

The effect of the bulges on a disk not only depend on its mass, but also on the bulge concentration. In this study we have not studied the observational effect of bulge concentration on our criterion because of different definitions of concentration in simulations (KD2018) and observations (Barway et al. 2016). A more detailed observational study that includes a wide sample containing barred and unbarred galaxies, is needed to test the effect of bulge mass and concentration in line with simulated results (KD2018).

\section{Summary}

The primary motivation for this study was to test the bar formation criterion given by KD2018 using an observational data set. We have made a small revision to the threshold value of $F B$ for 
bar formation in a galaxy disk. We find bars form in galaxy disks only if the $F B<0.13$ criterion is satisfied, where $\mathrm{FB}=\frac{G M_{\text {bulge }}}{2 R_{\mathrm{d}} V_{\text {tot }}^{2}}$. We applied the criterion to a sample of 103 barred galaxies for which the bulge, bar, and disk decompositions were given in the literature (Salo et al. 2015). We finally used 63 barred galaxies for the study, due to the constraints of matching the simulation models to observed galaxies and the availability of rotation curves. We find that $\approx 92 \%$ of all the barred galaxies in our sample follow the theoretical bar formation criterion, and the outliers are either interacting with companions or have patchy rotation curves. We also find that our criterion is a necessary but not a sufficient condition for bar formation as the effect of disk velocity dispersion cannot be fully accounted for in this criterion as it uses isolated galaxy simulations.

In future, this study can be improved by using precise rotation velocities at disk scale lengths using Integral Field Unit data, as such data can provide rotation velocities at all radii. This is especially important for galaxies that do not have a strong gas component and for which it is not possible to obtain $\mathrm{HI}$ or $\mathrm{CO}$ rotation curves. A much broader study of the bar formation criterion for disk galaxies carried out by calculating the $F B$ constant will improve our understanding of why bars form in some galaxies but not in all galaxies.

Acknowledgements. This research has made use of the VizieR catalog access tool, CDS, Strasbourg, France (DOI : 10.26093/cds/vizier). The original description of the VizieR service was published in 2000, A\&AS 143, 23. We acknowledge usage of the HyperLeda database (http://leda.univ-lyon1.fr). This research has also made use of the NASA/IPAC Extragalactic Database (NED), which is operated by the Jet Propulsion Laboratory, California Institute of Technology, under contract with the National Aeronautics and Space Administration. We also thank Christopher Mihos for the useful suggestion to add the value of the bar criterion for the Milky Way in this study. Finally, we thank the referee for useful comments that improved the content of this article.

\section{References}

Aguerri, J. A. L., Balcells, M., \& Peletier, R. F. 2001, A\&A, 367, 428 Aguerri, J. A. L., Méndez-Abreu, J., \& Corsini, E. M. 2009, A\&A, 495, 491 Athanassoula, E. 2005, MNRAS, 358, 1477

Athanassoula, E. 2008, MNRAS, 390, L69

Athanassoula, E., Lambert, J. C., \& Dehnen, W. 2005, MNRAS, 363, 496

Barazza, F. D., Jogee, S., \& Marinova, I. 2008, ApJ, 675, 1194

Barrera-Ballesteros, J. K., García-Lorenzo, B., Falcón-Barroso, J., et al. 2015, A\&A, 582, A21

Barway, S., Saha, K., Vaghmare, K., \& Kembhavi, A. K. 2016, MNRAS, 463, L41

Baugh, C. M., Cole, S., \& Frenk, C. S. 1996, MNRAS, 283, 1361

Beckmann, V., Gehrels, N., Shrader, C. R., \& Soldi, S. 2006, ApJ, 638, 642

Bennett, C. L., Larson, D., Weiland, J. L., et al. 2013, ApJS, 208, 20

Binney, J., \& Tremaine, S. 2008, Galactic Dynamics: second edition (Princeton: Princeton University Press)

Bland-Hawthorn, J., \& Gerhard, O. 2016, ARA\&A, 54, 529
Bournaud, F., Jog, C. J., \& Combes, F. 2007, A\&A, 476, 1179 Bovy, J., \& Rix, H.-W. 2013, ApJ, 779, 115

Combes, F., Debbasch, F., Friedli, D., \& Pfenniger, D. 1990, A\&A, 233, 82 Das, M., Teuben, P. J., Vogel, S. N., et al. 2003, ApJ, 582, 190

Das, M., Laurikainen, E., Salo, H., \& Buta, R. 2008, Ap\&SS, 317, 163 Debattista, V. P., Mayer, L., Carollo, C. M., et al. 2006, ApJ, 645, 209 Díaz-García, S., Salo, H., \& Laurikainen, E. 2016, A\&A, 596, A84 Efstathiou, G., Lake, G., \& Negroponte, J. 1982, MNRAS, 199, 1069 Eggen, O. J., Lynden-Bell, D., \& Sandage, A. R. 1962, ApJ, 136, 748 Elmegreen, B. G., Sundin, M., Kaufman, M., Brinks, E., \& Elmegreen, D. M. 1995, ApJ, 453, 139

Erwin, P., Saglia, R. P., Fabricius, M., et al. 2015, MNRAS, 446, 4039

Fisher, D. B., \& Drory, N. 2008, AJ, 136, 773

Gerhard, O. 2002, in The Galactic Bar, eds. G. S. Da Costa, E. M. Sadler, \& H. Jerjen, ASP Conf. Ser., 273, 73

Graham, A. W., \& Worley, C. C. 2008, MNRAS, 388, 1708

Guo, M., Du, M., Ho, L. C., Debattista, V. P., \& Zhao, D. 2020, ApJ, 888, 65

Heller, C. H., \& Shlosman, I. 1994, ApJ, 424, 84

Hohl, F. 1976, AJ, 81, 30

Hopkins, P. F., Cox, T. J., Younger, J. D., \& Hernquist, L. 2009, ApJ, 691, 1168

Hopkins, P. F., Bundy, K., Croton, D., et al. 2010, ApJ, 715, 202

Kataria, S. K., \& Das, M. 2018, MNRAS, 475, 1653

Kataria, S. K., \& Das, M. 2019, ApJ, 886, 43

Kauffmann, G., White, S. D. M., \& Guiderdoni, B. 1993, MNRAS, 264, 201

Kormendy, J., \& Kennicutt, R. C., Jr 2004, ARA\&A, 42, 603

Laurikainen, E., Salo, H., Buta, R., \& Knapen, J. H. 2007, MNRAS, 381, 401

Lelli, F., McGaugh, S. S., \& Schombert, J. M. 2016, AJ, 152, 157

Licquia, T. C., Newman, J. A., \& Bershady, M. A. 2016, ApJ, 833, 220

Makarov, D., Prugniel, P., Terekhova, N., Courtois, H., \& Vauglin, I. 2014, A\&A, 570, A13

Martinez-Valpuesta, I., Shlosman, I., \& Heller, C. 2006, ApJ, 637, 214

McGaugh, S. S. 2018, Res. Notes Am. Astron. Soc., 2, 156

McMillan, P. J. 2011, MNRAS, 414, 2446

Naab, T., Oser, L., Emsellem, E., et al. 2014, MNRAS, 444, 3357

Norman, C. A., Sellwood, J. A., \& Hasan, H. 1996, ApJ, 462, 114

Ostriker, J. P., \& Peebles, P. J. E. 1973, ApJ, 186, 467

Paturel, G., Theureau, G., Bottinelli, L., et al. 2003, A\&A, 412, 57

Peng, C. Y., Ho, L. C., Impey, C. D., \& Rix, H.-W. 2002, AJ, 124, 266

Peng, C. Y., Ho, L. C., Impey, C. D., \& Rix, H.-W. 2010, AJ, 139, 2097

Pfenniger, D., \& Norman, C. 1990, ApJ, 363, 391

Portail, M., Wegg, C., Gerhard, O., \& Martinez-Valpuesta, I. 2015, MNRAS, 448, 713

Qu, Y., Di Matteo, P., Lehnert, M. D., van Driel, W., \& Jog, C. J. 2011, A\&A, 535, A5

Raha, N., Sellwood, J. A., James, R. A., \& Kahn, F. D. 1991, Nature, 352, 411

Regan, M. W., \& Teuben, P. J. 2004, ApJ, 600, 595

Salo, H., Laurikainen, E., Laine, J., et al. 2015, ApJS, 219, 4

Sellwood, J. A. 1980, A\&A, 89, 296

Shen, J., \& Sellwood, J. A. 2004, ApJ, 604, 614

Sheth, K., Elmegreen, D. M., Elmegreen, B. G., et al. 2008, ApJ, 675, 1141

Skibba, R. A., Masters, K. L., Nichol, R. C., et al. 2012, MNRAS, 423, 1485

Sofue, Y., Tutui, Y., Honma, M., et al. 1999, ApJ, 523, 136

Telles, E., \& Terlevich, R. 1995, MNRAS, 275, 1

Thomas, H. C., Dunne, L., Clemens, M. S., et al. 2002, MNRAS, 329, 747

Toomre, A. 1981, in Structure and Evolution of Normal Galaxies, eds. S. M. Fall, \& D. Lynden-Bell, 111

Valluri, M. 1993, ApJ, 408, 57

Watkins, A. E., Mihos, J. C., Harding, P., \& Feldmeier, J. J. 2014, ApJ, 791, 38

Yurin, D., \& Springel, V. 2014, MNRAS, 444, 62 


\section{Appendix A: Table}

Table A.1. Galaxy samples used for bar formation criterion study.

\begin{tabular}{|c|c|c|c|c|c|c|}
\hline Galaxy name & Morphology & $\begin{array}{c}V_{\text {rot }} \\
\left(\mathrm{km} \mathrm{s}^{-1}\right) \\
\end{array}$ & $\begin{array}{c}\text { Bulge magnitude } \\
\text { (3.6 micron AB magnitude) }\end{array}$ & $\mathrm{B} / \mathrm{T}$ & $\begin{array}{l}\text { Inclination } \\
\text { (degrees) }\end{array}$ & $F B$ \\
\hline ESO 027-001 & $\mathrm{SBc}$ & 87.2 & 13.985 & 0.087 & 37 & 0.019 \\
\hline ESO 509-026 & $\mathrm{SABm}$ & 21.8 & 15.55 & 0.232 & 65.5 & 0.181 \\
\hline IC 4214 & $\mathrm{SBa}$ & 204.3 & 11.917 & 0.299 & 55.4 & 0.022 \\
\hline NGC 0210 & $\mathrm{SABb}$ & 154.2 & 11.965 & 0.291 & 55.4 & 0.009 \\
\hline NGC 0254 & SO-a & 148.7 & 12.428 & 0.333 & 57.2 & 0.016 \\
\hline NGC 0615 & $\mathrm{Sb}$ & 183.4 & 12.819 & 0.201 & 74 & 0.012 \\
\hline NGC 0936 & S0-a & 341.3 & 11.275 & 0.195 & 50.4 & 0.004 \\
\hline NGC 1015 & $\mathrm{Sa}$ & 120.7 & 13.508 & 0.209 & 35.5 & 0.017 \\
\hline NGC 1022 & $\mathrm{SBa}$ & 86.5 & 12.269 & 0.278 & 59.9 & 0.064 \\
\hline NGC 1232 & $\mathrm{SABc}$ & 185.2 & 13.814 & 0.022 & 32.7 & 0.001 \\
\hline NGC 1326 & $\mathrm{~S} 0-\mathrm{a}$ & 138.4 & 11.224 & 0.318 & 52.7 & 0.029 \\
\hline NGC 1367 & $\mathrm{Sa}$ & 232 & 12.382 & 0.138 & 51.9 & 0.008 \\
\hline NGC 1415 & $\mathrm{SO}-\mathrm{a}$ & 163.6 & 12.598 & 0.174 & 77.7 & 0.002 \\
\hline NGC 1533 & E-S0 & 132.7 & 11.697 & 0.249 & 64.8 & 0.083 \\
\hline NGC 2633 & $\mathrm{Sb}$ & 142.6 & 12.287 & 0.366 & 53.8 & 0.03 \\
\hline NGC 2750 & $\mathrm{SABc}$ & 86.4 & 13.884 & 0.148 & 45.4 & 0.007 \\
\hline NGC 2893 & $\mathrm{SO}-\mathrm{a}$ & 107.5 & 13.881 & 0.311 & 39.1 & 0.033 \\
\hline NGC 2962 & SO-a & 203.9 & 12.722 & 0.238 & 72.3 & 0.014 \\
\hline NGC 2968 & $\mathrm{Sa}$ & 122.9 & 11.905 & 0.363 & 53.3 & 0.044 \\
\hline NGC 3061 & $\mathrm{Sc}$ & 141.4 & 17.023 & 0.022 & 39.6 & 0.001 \\
\hline NGC 3166 & SO-a & 103.9 & 11.123 & 0.274 & 56.2 & 0.083 \\
\hline NGC 3254 & Sbc & 207.8 & 13.398 & 0.158 & 64.4 & 0.009 \\
\hline NGC 3266 & S0 & 264.5 & 13.811 & 0.248 & 34.7 & 0.005 \\
\hline NGC 3424 & $\mathrm{SBb}$ & 167.9 & 13.19 & 0.178 & 79.2 & 0.011 \\
\hline NGC 3489 & S0-a & 54.4 & 11.2 & 0.321 & 63.7 & 0.377 \\
\hline NGC 3507 & $\mathrm{SBb}$ & 117.9 & 13.754 & 0.068 & 31.9 & 0.006 \\
\hline NGC 3626 & S0-a & 190.4 & 11.939 & 0.297 & 55.8 & 0.018 \\
\hline NGC 3627 & $\mathrm{Sb}$ & 174.7 & 10.882 & 0.091 & 67.5 & 0.017 \\
\hline NGC 3684 & $\mathrm{Sbc}$ & 120.2 & 15.328 & 0.034 & 50.7 & 0.004 \\
\hline NGC 3686 & SBbc & 129.7 & 14.685 & 0.029 & 41.7 & 0.002 \\
\hline NGC 3705 & $\mathrm{SABa}$ & 167.9 & 13.012 & 0.091 & 72.2 & 0.016 \\
\hline NGC 3930 & $\mathrm{Sc}$ & 96.9 & 15.853 & 0.034 & 43 & 0.001 \\
\hline NGC 3953 & Sbc & 215.3 & 12.406 & 0.064 & 62.3 & 0.002 \\
\hline NGC 4045 & $\mathrm{Sa}$ & 169.1 & 12.586 & 0.245 & 55.9 & 0.019 \\
\hline NGC 4102 & $\mathrm{SABb}$ & 158 & 11.592 & 0.261 & 58.7 & 0.044 \\
\hline NGC 4133 & $\mathrm{SABb}$ & 166.1 & 13.904 & 0.147 & 51.3 & 0.01 \\
\hline NGC 4267 & E-S0 & 60.2 & 11.638 & 0.29 & 57.4 & 0.174 \\
\hline NGC 4319 & SBab & 112.5 & 13.758 & 0.099 & 72.5 & 0.013 \\
\hline NGC 4355 & SABa & 47 & 13.83 & 0.316 & 68.1 & 0.16 \\
\hline NGC 4569 & $\mathrm{Sab}$ & 185.8 & 11.222 & 0.146 & 70.9 & 0.023 \\
\hline NGC 4606 & $\mathrm{SBa}$ & 70.2 & 13.608 & 0.17 & 62.7 & 0.009 \\
\hline NGC 4795 & $\mathrm{SBa}$ & 191.8 & 13.533 & 0.149 & 53.6 & 0.01 \\
\hline NGC 4897 & Sbc & 168.1 & 13.726 & 0.2 & 38.3 & 0.011 \\
\hline NGC 4984 & SO-a & 125.3 & 11.092 & 0.382 & 47.1 & 0.041 \\
\hline NGC 5005 & $\mathrm{SABb}$ & 251 & 11 & 0.152 & 77.1 & 0.023 \\
\hline NGC 5195 & $\mathrm{SBa}$ & 120.3 & 10.122 & 0.253 & 40.5 & 0.024 \\
\hline NGC 5205 & SBbc & 136.5 & 13.734 & 0.287 & 56 & 0.015 \\
\hline NGC 5377 & $\mathrm{Sa}$ & 177.1 & 11.732 & 0.394 & 77.2 & 0.017 \\
\hline
\end{tabular}

Note: Galaxy morphology, $V_{\text {rot }}$, and inclination angle are taken from the Hyperleda database. The bulge magnitude and bulge-to-total mass ratio is taken from Salo et al. (2015). 
S. K. Kataria et al.: Testing a theoretical prediction for bar formation in galaxies with bulges

Table A.1. continued.

\begin{tabular}{lcccccc}
\hline \hline Galaxy name & Morphology & $\begin{array}{c}V_{\text {rot }} \\
\left(\mathrm{km} \mathrm{s}^{-1}\right)\end{array}$ & $\begin{array}{c}\text { Bulge magnitude } \\
\text { (3.6 micron AB magnitude) }\end{array}$ & B/T & $\begin{array}{c}\text { Inclination } \\
\text { (degrees) }\end{array}$ & $F B$ \\
\hline NGC 5443 & $\mathrm{Sb}$ & 167.6 & 13.608 & 0.144 & 77.4 & 0.007 \\
NGC 5448 & $\mathrm{Sa}$ & 208 & 12.517 & 0.282 & 64.4 & 0.009 \\
NGC 5473 & $\mathrm{E}-\mathrm{S} 0$ & 158 & 12.299 & 0.276 & 47.7 & 0.043 \\
NGC 5566 & $\mathrm{SBab}$ & 204 & 11.511 & 0.228 & 75.6 & 0.01 \\
NGC 5713 & $\mathrm{SABb}$ & 107.9 & 13.571 & 0.069 & 48.2 & 0.025 \\
NGC 5728 & $\mathrm{Sa}$ & 224.4 & 11.934 & 0.263 & 53.1 & 0.008 \\
NGC 5878 & $\mathrm{Sb}$ & 214.5 & 12.795 & 0.148 & 73.8 & 0.007 \\
NGC 6278 & $\mathrm{S} 0$ & 76 & 13.007 & 0.277 & 78.8 & 0.151 \\
NGC 6923 & $\mathrm{SBb}$ & 191.1 & 13.334 & 0.137 & 64.2 & 0.008 \\
NGC 7280 & $\mathrm{S} 0-\mathrm{a}$ & 129.4 & 13.23 & 0.223 & 58.7 & 0.012 \\
NGC 7465 & $\mathrm{S} 0$ & 95 & 13.092 & 0.357 & 64.2 & 0.113 \\
NGC 7479 & $\mathrm{SBbc}$ & 238.3 & 12.958 & 0.083 & 43 & 0.004 \\
NGC 7531 & $\mathrm{SABb}$ & 161.2 & 13.115 & 0.138 & 68.9 & 0.011 \\
NGC 7582 & $\mathrm{SBab}$ & 194.7 & 11.185 & 0.252 & 68 & 0.016 \\
NGC 7731 & $\mathrm{SBa}$ & 92.7 & 14.839 & 0.203 & 45.5 & 0.019 \\
\hline
\end{tabular}

